\title{
Neutrophil-lymphocyte and Platelet-lymphocyte Ratios among Adolescents with Substance Use Disorder: A Preliminary Study
}

\author{
Serdar Karatoprak', Necati Uzun², Mehmet Akif Akıncı', Yunus Emre Dönmez ${ }^{4}$ \\ ${ }^{1}$ Department of Child and Adolescent Psychiatry, Elazıg Fethi Sekin City Hospital, Elazıg, ${ }^{2}$ Department of Child and Adolescent Psychiatry, \\ Meram School of Medicine, Necmettin Erbakan University, Konya, ${ }^{3}$ Department of Child and Adolescent Psychiatry, Dr. Ali Kemal Belviranlı \\ Maternity and Children's Hospital, Konya, ${ }^{4}$ Department of Child and Adolescent Psychiatry, School of Medicine, Inonu University, Malatya, \\ Turkey
}

Objective: Substance use disorder (SUD) is a serious public health problem affecting both the individual and the society, and substance use-related deaths and disability have been shown to increase gradually. Recent etiologic studies have reported that there is a relation between inflammatory parameters and psychiatric disorders. The neutrophil-lymphocyte ratio (NLR) and platelet-lymphocyte ratio (PLR) used as an indicator of inflammation have been shown to be increased in various psychiatric disorders. In this study, we aimed to investigate the NLR and PLR in adolescents with SUD. Methods: This study was conducted by retrospectively examining the records of 55 male adolescents who were followed up with SUD in a child and adolescent psychiatry outpatient clinic between November 2019-June 2020. Patients who had comorbid psychiatric disorder were included, and those who received any psychotropic medication were excluded. A total of 61 healthy male adolescents in the same age range without any psychiatric disorders were recruited as a control group. Neutrophil-lymphocyte-platelet counts were noted retrospectively from complete blood tests, and NLR-PLR were calculated.

Results: The NLR and PLR of adolescents with SUD were significantly higher than the healthy adolescents $(p<0.01$, $p<0.01$, respectively). In addition, conduct disorder, depression, and attention deficit/hyperactivity disorder, which were determined as the most common comorbid psychiatric disorders had no effects on NLR $(p=0.513, p=0.584$, $p=0.394$, respectively) and $\operatorname{PLR}(p=0.210, p=0.346, p=0.359$, respectively).

Conclusion: The results of current study indicate that inflammatory processes may have a key role in the pathophysiology of SUD.

KEY WORDS: Substance use disorder; Inflammation; Neutrophil; Lymphocyte; Platelet; Adolescents.

\section{INTRODUCTION}

Substance use disorder (SUD), involving cannabis, opioids, cocaine, and amphetamines, is a major public health issue that affects both individuals and communities [1]. The 2019 report of the United Nations Office for Drugs and Crime states that deaths and disability-adjusted life years related to drug use have nearly doubled in the last 30 years and that $5.5 \%$ of individuals globally had

Received: August 22, 2020 / Revised: October 25, 2020

Accepted: November 9, 2020

Address for correspondence: Serdar Karatoprak

Department of Child and Adolescent Psychiatry, Elazıg Fethi

Sekin City Hospital, Ulukent Mahallesi (Doğukent Depo Mevkii)

Onbașı Sokak No: 99, Elazıg, Turkey

E-mail: srdrkrtprk@hotmail.com

ORCID: https://orcid.org/0000-0001-6319-8948 used drugs at least once in the previous year was reported [2]. It was estimated that globally, 12.6 million students aged 15-16 used any drugs in 2017 [2].

Once-off substance use is not considered to constitute substance addiction. Substance abuse usually begins with adolescents subject to peer effects trying drugs for the first time. In some, this escalates into maintenance of a drug habit, abuse, and, eventually, addiction [3]. Many risk factors for substance abuse have been identified; however, the pathophysiology of addiction has not yet been fully understood [3]. Recent studies have shown that immune dysregulation in proinflammatory and regulatory processes may play a role in addiction. Bowers and Kalivas [4] and Kalivas et al. [5] found that after 3 weeks of acute cocaine administration, expression of the glial fibril-

(c) This is an Open-Access article distributed under the terms of the Creative Commons Attribution Non-Commercial License (http://creativecommons.org/licenses/by-nc/4.0) which permits unrestricted non-commercial use, distribution, and reproduction in any medium, provided the original work is properly cited. 
lary acidic protein and vimentin 3 increased. These proteins facilitate neuronal migration, modulate the formation of synapses, and regulate synaptic strength. In a study of 10 chronic cocaine abusers and nine controls, Little et al. [6] found that the density of activated macrophages and microglia was greater in the midbrains of abusers than in the midbrains of controls. Similarly, opioids have been found to affect the immune system directly by affecting macrophages and lymphocytes and indirectly by causing changes in central nervous system that cause neurotoxicity. Raghavendra et al. [7] found substantial increases in glial cell over-activation and levels of proinflammatory cytokines, such as IL-1 $\beta$, TNF- $\alpha$, and IL-6, in a neuropathic rodent model of chronic morphine treatment. A significantly increased neutrophil count and a significant reduction in lymphocytes have also been found in heroin addicts $[8,9]$. These results suggest a possible relationship between psychostimulant and opioid use and inflammatory processes.

The inflammatory process is complex and regulated by a number of soluble molecules such as cytokines, chemokines, and acute phase proteins [10]. It has been determined that patients with psychiatric disorders such as major depressive disorder (MDD), schizophrenia (SZ), and bipolar disorder (BD) have increased levels of proinflammatory immune markers and that an activated inflammatory system has a key role in these disorders [11-13]. Although proinflammatory cytokines are produced in the peripheral system, they have been shown to affect the immune cells and mediators in the central nervous system and increase inflammatory responses in the central nervous system [10]. In addition, elevated levels of proinflammatory cytokines have been found in the cerebrospinal fluid and postmortem brain tissue of patients with MDD and SZ [10].

Many studies on the relationship between psychiatric disorders and inflammation have used the neutrophil-lymphocyte ratio (NLR) and the platelet-lymphocyte ratio $(P L R)$ as parameters to indicate inflammation because they can be easily and cost-effectively obtained from a complete blood count analysis. It has been found that NLR, an indicator of subclinical inflammation, is associated with psychiatric disorders including SZ, MDD, $\mathrm{BD}$, and obsessive compulsive disorder (OCD) [8,14-16]. In cases of systemic inflammation, platelets also increase in number due to endothelial damage. Like NLR, PLR is an index of inflammatory balance; it has also been found to increase in individuals with psychiatric disorders such as MDD, BD, and SZ $[17,18]$.

To date, no study has evaluated NLR and PLR in adolescents with SUD. The aim of the current study was to examine immune system dysregulation in adolescents with SUD by assessing NLR and PLR. We hypothesized that NLR and PLR would be higher in adolescents with SUD than in healthy controls.

\section{METHODS}

\section{Participants}

This study was conducted with 55 male adolescents aged 12-18 with SUD and 61 healthy male adolescents in the same age range. Psychiatric evaluation of all participants was carried out using the 5th edition of the Diagnostic and Statistical Manual of Mental Disorders and psychiatric interviews was based on the Schedule for Affective Disorders and Schizophrenia for School-Age Children-Present and Lifetime Version-Turkish Adaptation $[19,20]$. Patients who had comorbid psychiatric disorders were included in the study. However, those who had received any psychotropic medication were excluded.

Records of all participants were examined to assess their physical health based on clinical examinations and laboratory tests. The exclusion criteria for both groups were as follows: obesity (body mass index $>30 \mathrm{~kg} / \mathrm{m}^{2}$ ), presence of acute or chronic medical disorder, including acute or chronic inflammatory disease, concomitant use of any medications, abnormal vital signs (e.g., fever) or laboratory test results (e.g., showing anemia, leukopenia). In the study, blood samples taken routinely from the patients after 12 hours of fasting in the outpatient follow-up were used. For both SUD group and control group, NLR was obtained by dividing the absolute value of peripheral neutrophil count by lymphocyte count based on standard laboratory panel data. Similarly, PLR was obtained by dividing the absolute value of platelet count by lymphocyte count based on standard laboratory panel data.

The study was approved by the Firat University NonInterventional Research Ethics Committee (Report number: 2020/11-11) and conducted in accordance with the principles of the Helsinki Declaration. 


\section{Statistical Analysis}

The statistical analyses were conducted using the SPSS version 22.0 for Windows software package (IBM Co., Armonk, NY, USA). The categorical variables of the participants were presented using number and percentage values. Chi-square test was used to compare categorical variables, and the Kolmogorov - Smirnov test was used to evaluate the normality of the data distributions. Independent samples $t$ test was used to compare normally distributed variables, and the Mann - Whitney $U$ test was used to compare variables that were not normally distributed. The potential predictive effects of variables on NLR and PLR were evaluated by stepwise linear regression analysis. Before the regression analysis, variables that did not display normal distribution were log-transformed. A value of $p<0.05$ (two-tailed) was considered to indicate significance. Bonferroni correction was used to obtain the significance level.

\section{RESULTS}

The mean ages of the SUD sample $(n=55)$ and control group $(n=61)$ were determined as $16(\% 50=16)$ and 16 $(\% 50=16)$ years, respectively. There was no significant difference between the SUD and control groups in terms of age $(Z=-0.85, p=0.392)$. The mean age of substance

Table 1. The characteristics of the adolescents with substance use disorder regarding substance use

\begin{tabular}{llc}
\hline \multicolumn{1}{c}{ Variable } & & \multicolumn{1}{c}{ Value } \\
\hline Cigarette using & No & $8(14.5)$ \\
& Yes & $47(85.5)$ \\
Benzodiazepine using & No & $50(90.9)$ \\
Heroin using & Yes & $5(9.1)$ \\
& No & $44(80)$ \\
Bonsai using & Yes & $11(20)$ \\
& No & $46(83.6)$ \\
Volatile substance using & Yes & $9(16.4)$ \\
& No & $38(69.1)$ \\
Ecstasy using & Yes & $17(30.9)$ \\
\multirow{2}{*}{ Cannabis using } & No & $27(49.1)$ \\
& Yes & $28(50.9)$ \\
More than one substance using & No & $13(23.6)$ \\
& Yes & $42(76.4)$ \\
Onset age of substance use & No & $14(25.5)$ \\
Duration of substance use & & $41(74.5)$ \\
\hline
\end{tabular}

Values are presented as number (\%) or mean \pm standard deviation. use onset was $13.82 \pm 1.40$ years and the mean duration of the disorder at the time of the study was $2.83 \pm 1.34$ years. Approximately $75 \%(n=41)$ of the cases in the SUD sample had used more than one substance. The most frequently used substances were cannabis $(76.4 \%)$, ecstasy $(50.9 \%)$, volatile substances $(30.9 \%)$, and heroin $(20 \%)$. In addition, $85.5 \%$ of the cases used cigarettes. The characteristics of the adolescents with SUD regarding substance use are presented in Table 1.

Of the cases diagnosed with SUD, $65.5 \%(n=36)$ of the cases had at least one comorbid psychiatric disorder. The most common comorbid psychiatric disorders were conduct disorder (CD) $(n=23)$, MDD $(n=20)$, and attention deficit/hyperactivity disorder (ADHD) $(n=9)$. The data on comorbid psychiatric disorders are presented in Table 2.

As Table 3 shows, the neutrophil count was higher in adolescents with SUD (median 4.09; interquartile range [IQR] 3.34-5.23) than in the control group (median 3.63; IQR 3.18-4.40), and the lymphocyte count was lower in adolescents with SUD (median 2.38; IQR 2.002.98) than in the control group (median 2.68; IQR 2.333.16). The platelet count was similar in both groups (median 258 [226-303] in adolescents with SUD and median 262 [222-287] in the control group). There was no statistically significant difference between the groups in terms of neutrophil, platelet and the lymphocyte counts.

The median NLR was 1.65 for the SUD participants and 1.44 for the healthy controls. The median PLR was found 111.05 for the SUD participants and 92.39 for the healthy controls. The results of the Mann-Whitney $U$ test showed that both NLR and PLR were significantly higher in the adolescents with SUD than in the control group ( $p=$ $0.006, p=0.007$, respectively). The effects of various variables on NLR and PLR were evaluated by stepwise linear regression analysis. Log transformation was performed to

Table 2. Comorbid psychiatric disorders of adolescents with substance use disorder

\begin{tabular}{lc}
\hline \multicolumn{1}{c}{ Comorbid psychiatric disorder } & Value \\
\hline Conduct disorder & $23(41.8)$ \\
Depressive disorder & $20(36.4)$ \\
Attention deficit hyperactivity disorder & $9(16.4)$ \\
Anxiety disorders & $3(5.4)$ \\
Psychotic disorder & $2(3.6)$ \\
\hline
\end{tabular}

Values are presented as number (\%). 
Table 3. Comparison of blood count parameters and age between adolescents with substance use disorder group and healthy controls

\begin{tabular}{lcccc}
\hline Variable & SUD group & Control group & $Z$ or $F$ & $p$ value \\
\hline Age & $17(16-17)$ & $17(16-17)$ & -0.85 & 0.392 \\
PLT & $258(226-303)$ & $262(222-287)$ & -0.36 & 0.715 \\
NEU & $4.09(3.34-5.23)$ & $3.63(3.18-4.40)$ & -1.86 & 0.062 \\
LYM & $2.38(2.00-2.98)$ & $2.68(2.33-3.16)$ & -2.34 & 0.019 \\
NLR & $1.65(1.17-2.18)$ & $1.44(1.10-1.68)$ & -2.73 & 0.006 \\
PLR & $111.05(86.11-130.62)$ & $92.39(83.75-107.28)$ & -2.67 & 0.007 \\
Lnx NLR & $0.52 \pm 0.45$ & $0.30 \pm 0.27$ & 8.13 & 0.005 \\
Lnx PLR & $4.67 \pm 0.35$ & $4.55 \pm 0.24$ & 7.15 & 0.009 \\
\hline
\end{tabular}

Values are presented as median (interquartile range) or mean \pm standard deviation.

PLT, platelete; NEU, neutrophile; LYM, lymphocyte; NLR, Neutrophil-lymphocyte ratio; PLR, platelet-lymphocyte ratio.

Lnx NLR and Lnx PLR were compared with independent sample $t$ test, and Mann - Whitney $U$ test was used for other variables. For independent sample $t$ test, bonferroni corrected significance of $p \leq 0.025(0.05 / 2=0.025)$. For Mann - Whitney $U$ test, bonferroni corrected significance of $p \leq$ $0.025(0.05 / 2=0.025), p \leq 0.008(0.05 / 6=0.008)$.

Table 4. Stepwise linear regression analysis for Lnx NLR and Lnx PLR

\begin{tabular}{lrrrrr}
\hline \multirow{2}{*}{ Variable } & \multicolumn{2}{c}{ Lnx NLR } & & \multicolumn{2}{c}{ Lnx PLR } \\
\cline { 2 - 3 } \cline { 5 - 6 } & \multicolumn{1}{c}{$\beta$} & \multicolumn{1}{c}{$p$ value } & \multicolumn{1}{c}{$\beta$} & $p$ value \\
\hline Groups (SUD) & 0.274 & 0.003 & & 0.217 & 0.020 \\
Age & 0.080 & 0.377 & -0.044 & 0.638 \\
Duration of the disorder & 0.059 & 0.726 & -0.036 & 0.832 \\
Depressive disorder & -0.057 & 0.584 & -0.099 & 0.346 \\
Conduct disorder & -0.070 & 0.513 & & 0.136 & 0.210 \\
ADHD & -0.081 & 0.394 & & 0.089 & 0.359 \\
\hline
\end{tabular}

SUD, substance use disorder; ADHD, attention deficit hyperactivity disorder.

Adjusted $R^{2}$ for $\operatorname{Ln} x$ NLR $=0.067$; Adjusted $R^{2}$ for Lnx PLR $=0.039$.

ensure normal distribution before regression. The logtransformed NLR and PLR values were examined by an independent samples $T$ test before regression, and both were found to be significantly higher in the adolescents with SUD than in the control group ( $p=0.005, p=0.009$, respectively). Substance use was found to have a potential predictive effect on the log-transformed NLR and PLR values. However, no potential predictive effects of the duration of SUD or the presence of the comorbid conditions $\mathrm{CD}, \mathrm{MDD}$, and $\mathrm{ADHD}$ on log-transformed NLR $(p=$ $0.726, p=0.513, p=0.584, p=0.394$, respectively) or log-transformed PLR ( $p=0.832, p=0.210, p=0.346, p=$ 0.359 , respectively) were detected in the regression analysis (Table 4).

\section{DISCUSSION}

The current study examined whether NLR and PLR were related to SUD in adolescents. The results show stat- istically significant difference in NLR and PLR between adolescents with SUD and healthy controls. To our knowledge, this is the first study to investigate NLR and PLR in adolescents with SUD.

Neutrophils, lymphocytes, and platelets have an important role in controlling inflammation, and systemic inflammation is associated with the blood levels of these cells (i.e., neutrophilia, lymphopenia, and thrombocytosis) [21]. Neutrophils are the first responders in the immune defense system; they demonstrate phagocytic and apoptotic activity via synthesis of proinflammatory molecules [22]. Therefore, it has been suggested that neutrophil counts are an important indicator of chronic inflammation [23]. By contrast, lymphocytes play an important role in regulating, controlling, and inhibiting the inflammatory process. In conclusion, NLR is important for evaluating the inflammatory process as it provides information about neutrophils, which are responsible for inflammation progression, and lymphocytes, which are responsible for inflammation regulation [21]. Platelets, which regulate endothelial permeability and the recruitment of neutrophils and macrophages, are another firstline inflammatory marker [24]. It has been determined that increased platelet levels are closely associated with proinflammatory molecules and acute phase reactants [25]. The high platelet count contributes to increasing the destructive effect of the proinflammatory process [26]. Hence, PLR is another marker of the inflammatory process.

Previous studies have found associations between both NLR and PLR and several psychiatric disorders, including SZ, BD, MDD, and OCD $[8,16,27,28]$. Özyurt and Binici [16] determined that the NLR and PLR of adolescent pa- 
tients with OCD were higher than in adolescents with no psychiatric disorders. In addition, they found that the NLR was higher in adolescents with OCD and comorbid anxiety disorder than in either healthy controls or adolescents with pure OCD. Previous studies investigating the relationship between manic episodes and NLR in patients with BD found that NLR was increased in patients with manic episodes [27-29]. In their study, conducted with 61 manic and 55 euthymic patients and 54 control cases, Kalelioglu et al. [28] found that NLR and PLR were significantly higher in both manic and euthymic bipolar patients than in the control group. Similarly, in a study of 41 MDD patients, Demir et al. [30] determined that NLR was high in patients with depression.

Studies investigating inflammatory markers in patients with addiction have shown that levels of cytokines, acute phase reactants, and immunoglobulin G-M are higher in addicted individuals than healthy controls. In a study conducted with 34 heroin-addicted patients receiving methadone treatment, Chan et al. [31] found increased production of IL-1 $\beta$, IL-6, and IL-8 in the group of addicted patients. In addition, Chen et al. [32] found that the plasma levels of TNF- $\alpha$ and IL-8 were significantly higher in heroin addicts than in healthy controls. Cicek et al. [23], in their study with 90 male patients with heroin addiction, also found that mean NLR and PLR and neutrophil and platelet counts were significantly higher in heroin-addicted patients than in healthy controls. Another study conducted with 107 patients with cocaine use disorder revealed that NLR was significantly higher in samples from cocaine users than in those from non-cocaine users [33]. Similar to these previous studies, the current study found that NLR and PLR were significantly higher in adolescents with SUD than in healthy controls. However, $65 \%$ of adolescents with SUD included in this study also had comorbid psychiatric disorders. As explained above, many psychiatric disorders have been associated with high NLR and PLR. Although no studies on the relationship between NLR and CD, which was the most common comorbid psychiatric disorder in this study, could be found, NLR and PLR have been found to be high in individuals with MDD and ADHD [30,34]. A regression analysis was carried out to evaluate a potential predictive effect of comorbid psychiatric disorders on the log-transformed NLR and PLR values; no potential predictive effect was determined. These results show that the between-group differences in NLR and PLR were due to substance use. Although neutrophil and platelet counts were higher in adolescents with SUD compared to the control group, no statistical difference was found between the groups. A possible reason for this may be that the duration of substance use is generally shorter in adolescents than in adults. Therefore, we propose that NLR and PLR can be used as a marker of inflammation in the early stage of SUD.

Inflammation caused by substances in the brain may adversely affect the neuronal development of the brain during adolescence, leading to impaired self-regulation, impulse control, and executive decision-making abilities. Such impairment in this developmental stage may also increase the risk of substance addiction. Indeed, Pumariega et al. [35] reported that the onset of substance use early in life is linked to a greater risk of substance dependence in later periods of life. Another major problem in adolescents is multi-drug use, which leads to greater frequency of substance use [36]. This may be due to the fact that multiple substance use causes more inflammation and, hence, has a greater negative effect on brain development. Another finding that indicates a possible role of inflammatory dysregulation in addiction is that of a gender difference in both the development of addiction and the severity of its symptoms and consequences. It has been reported that addiction development is faster and negative consequences and addiction symptoms more severe in females than in males [37-39]. These results may be due to the difference in gender differences in inflammatory responses. Females have been shown to produce a stronger inflammatory response than males [40], which may explain why addiction is more severe in females. Because of this stronger inflammatory response, we expect that females with SUD may also have higher NLR and PLR. Moreover, it has been reported that minocycline, a second-generation tetracycline that has powerful anti-inflammatory and neuroprotective properties, reduced neurotoxicity in the hippocampus and striatum by inhibiting microglial activation aroused after repeated administration of ecstasy and methamphetamine [41,42]. Attarzadeh-Yazdi et al. [41] determined that in rats, daily minocycline injections during the extinction period blocked the persistence of the methamphetamine rewarding effect. In addition, a study carried out with healthy participants found that minocycline reduces the sub- 
jective rewarding effects of dextroamphetamine [43]. It has been suggested that these positive effects of minocycline occur via its effects on dopamine and glutamate transmission in the striatum and its inhibitory effect on microglial activation and neurotoxicity, the nitric oxide synthase enzyme, and the p38 mitogen-activated protein kinase signaling pathway $[41,43]$. These results suggest that early prevention of inflammation in adolescents who use substances may prevent the development of substance addiction in later periods.

However, this is the first study to investigate NLR and PLR in adolescents with SUD, and it has certain limitations. The primary limitation of our study is that the cross-sectional and retrospective nature of the study does not enable us to examine the continuity of the results. The secondly limitation is the small sample size, which does not allow generalization of the study results. Other limitations of the study were that all of the participants were males, that the participants had comorbid psychiatric disorders, that other inflammatory markers such as proinflammatory cytokines and acute phase reactants were not evaluated, and that participants could not be evaluated according to the type of substance they used because of the small sample size.

In conclusion, this study shows that NLR and PLR levels were statistically significantly higher in adolescents with SUD compared to healthy adolescents, which indicates that inflammatory dysregulation may have a key role in the pathophysiology of SUD in adolescents. If inflammatory dysregulation is one of the underlying mechanisms in the development of SUD, anti-inflammatory agents may be a promising treatment option for substance abuse. Further longitudinal studies with larger samples including male and female subjects are needed to elucidate the association between SUD and high NLR and PLR.

\section{- Conflicts of Interest}

No potential conflict of interest relevant to this article was reported.

\section{Author Contributions}

Conceptualization: Serdar Karatoprak, Necati Uzun. Data acquisition: Serdar Karatoprak, Necati Uzun, Mehmet Akif Akıncı. Statistic: Yunus Emre Dönmez, Mehmet Akif Akıncı. Writing - original draft: Serdar Karatoprak, Necati Uzun, Mehmet Akif Akıncı, Yunus Emre Dönmez. Writing - review \& editing: Serdar Karatoprak, Necati Uzun, Mehmet Akif Akıncı, Yunus Emre Dönmez.

\section{ORCID}

Serdar Karatoprak https://orcid.org/0000-0001-6319-8948 Necati Uzun https://orcid.org/0000-0003-3381-2331

Mehmet Akif Akıncı https://orcid.org/0000-0002-6436-4394 Yunus Emre Dönmez

https://orcid.org/0000-0002-7785-2805

\section{REFERENCES}

1. Çiftçi Demirci A, Erdoğan A, Yalçın Ö, Yıldızhan E, Koyuncu $\mathrm{Z}$, Eseroğlu T, et al. Sociodemographic characteristics and drug abuse patterns of adolescents admitted for substance use disorder treatment in Istanbul. Am I Drug A/cohol Abuse 2015:41:212-219.

2. United Nations Office on Drugs and Crime. World drug report 2019-global overview of drug demand and supply. New York:United Nations Publications;2019.

3. Roberts NJ, Fishbein D. An integrative perspective on the etiology of substance use. In: Sloboda Z, Petras H, Robertson E, Hingson $R$, editors. Prevention of substance use. Cham: Springer;2019. p.37-55.

4. Bowers MS, Kalivas PW. Forebrain astrog/ial plasticity is induced following withdrawal from repeated cocaine administration. Eur J Neurosci 2003;17:1273-1278.

5. Kalivas PW, McFarland K, Bowers S, Szumlinski K, Xi ZX, Baker D. Glutamate transmission and addiction to cocaine. Ann N Y Acad Sci 2003;1003:169-175.

6. Little KY, Ramssen E, Welchko R, Volberg V, Roland CJ, Cassin B. Decreased brain dopamine cell numbers in human cocaine users. Psychiatry Res 2009;168:173-180.

7. Raghavendra V, Tanga F, DeLeo JA. Inhibition of microg/ial activation attenuates the development but not existing hypersensitivity in a rat model of neuropathy. I Pharmacol Exp Ther 2003;306:624-630.

8. Guzel D, Yazici AB, Yazici E, Erol A. Evaluation of immunomodulatory and hematologic cell outcome in heroin/opioid addicts. J Addict 2018;2018:2036145.

9. Haghpanah T, Afarinesh M, Divsalar K. A review on hematological factors in opioid-dependent people lopium and heroin) after the withdrawal period. Addict Health 2010;2:9-16.

10. Debnath M, Doyle K, Langan C, McDonald C, Leonard B, Cannon D. Recent advances in psychoneuroimmunology: inflammation in psychiatric disorders. Trans/ Neurosci 2011;2. 121-137.

11. Myint AM, Leonard BE, Steinbusch HW, Kim YK. Th1, Th2, and Th3 cytokine alterations in major depression. I Affect Disord 2005;88:167-173.

12. Coelho FM, Reis HJ, Nicolato R, Romano-Silva MA, Teixeira $\mathrm{MM}$, Bauer ME, et al. Increased serum levels of inflammatory markers in chronic institutionalized patients with schizophrenia. 
Neuroimmunomodulation 2008;15:140-144.

13. Kim YK, Myint AM, Verkerk R, Scharpe S, Steinbusch H, Leonard B. Cytokine changes and tryptophan metabolites in medication-naïve and medication-free schizophrenic patients. Neuropsychobiology 2009;59:123-129.

14. Aydin Sunbul E, Sunbul M, Yanartas O, Cengiz F, Bozbay M, Sari I, et al. Increased neutrophil/lymphocyte ratio in patients with depression is correlated with the severity of depression and cardiovascular risk factors. Psychiatry Investig 2016;13. 121-126.

15. Semiz M, Yildirim O, Canan F, Demir S, Hasbek E, Tuman TC, et al. Elevated neutrophil/lymphocyte ratio in patients with schizophrenia. Psychiatr Danub 2014;26:220-225.

16. Özyurt G, Binici NC. The neutrophil-lymphocyte ratio and platelet-lymphocyte ratio in adolescent obsessive-compulsive disorder: does comorbid anxiety disorder affect inflammatory response? Psychiatry Res 2019;272:311-315.

17. Qin B, Ma N, Tang Q, Wei T, Yang M, Fu H, et al. Neutrophil to lymphocyte ratio $(N L R)$ and platelet to lymphocyte ratio $(P L R)$ were useful markers in assessment of inflammatory response and disease activity in SLE patients. Mod Rheumatol 2016;26:372-376.

18. Kayhan F, Gündüz Ş, Ersoy SA, Kandeğer A, Annagür BB. Relationships of neutrophil-lymphocyte and platelet-lymphocyte ratios with the severity of major depression. Psychiatry Res 2017;247:332-335.

19. American Psychiatric Association. Diagnostic and statistical manual of mental disorders: DSM-5. Arlington:American Psychiatric Association;2013.

20. Ünal F, Öktem F, Çetin Çuhadaroğlu F, Çengel Kültür SE, Akdemir D, Foto Özdemir D, et al. [Reliability and validity of the Schedule for Affective Disorders and Schizophrenia for School-Age Children-present and Lifetime Version, DSM-5 November 2016-Turkish adaptation (K-SADS-PL-DSM-5-T)]. Turk Psikiyatri Derg 2019;30:42-50. Turkish.

21. Lee YH, Song GG. Neutrophil-to-lymphocyte ratio, mean platelet volume and platelet-to-lymphocyte ratio in Behçet's disease and their correlation with disease activity: a metaanalysis. Int I Rheum Dis 2018;21:2180-2187.

22. Mayadas TN, Cullere X, Lowell CA. The multifaceted functions of neutrophils. Annu Rev Pathol 2014;9:181-218.

23. Cicek E, Demırel B, Cicek IE, Kıraç AS, Eren I. Increased neutrophil-lymphocyte and platelet-lymphocyte ratios in male heroin addicts: a prospective controlled study. Clin Psychopharmacol Neurosci 2018;16:190-196.

24. Dietrich-Muszalska A, Wachowicz B. Platelet haemostatic function in psychiatric disorders: effects of antidepressants and antipsychotic drugs. World I Biol Psychiatry 2017;18: 564-574.

25. Alexandrakis MG, Passam FH, Moschandrea IA, Christophoridou AV, Pappa CA, Coulocheri SA, et al. Levels of serum cytokines and acute phase proteins in patients with essential and cancer-related thrombocytosis. Am J Clin Oncol 2003;26:135-
140.

26. Balta S, Ozturk C. The platelet-lymphocyte ratio: a simple, inexpensive and rapid prognostic marker for cardiovascular events. Platelets 2015;26:680-681.

27. Özdin S, Sarisoy G, Böke Ö. A comparison of the neutrophillymphocyte, platelet-lymphocyte and monocyte-lymphocyte ratios in schizophrenia and bipolar disorder patients - a retrospective file review. Nord I Psychiatry 2017;71:509-512.

28. Kalelioglu T, Akkus M, Karamustafalioglu N, Genc A, Genc ES, Cansiz A, et al. Neutrophil-lymphocyte and platelet-lymphocyte ratios as inflammation markers for bipolar disorder. Psychiatry Res 2015;228:925-927.

29. Çakır U, Tuman TC, Yıldırım O. Increased neutrophil/lymphoctye ratio in patients with bipolar disorder: a preliminary study. Psychiatr Danub 2015;27:180-184.

30. Demir S, Atli A, Bulut M, İbiloğlu AO, Güneş M, Kaya MC, et al. Neutrophil-lymphocyte ratio in patients with major depressive disorder undergoing no pharmacological therapy. Neuropsychiatr Dis Treat 2015;11:2253-2258.

31. Chan YY, Yang SN, Lin JC, Chang JL, Lin JG, Lo WY. Inflammatory response in heroin addicts undergoing methadone maintenance treatment. Psychiatry Res 2015;226:230234.

32. Chen SL, Lee SY, Tao PL, Chang YH, Chen $\mathrm{SH}$, Chu $\mathrm{CH}$, et al. Dextromethorphan attenuated inflammation and combined opioid use in humans undergoing methadone maintenance treatment. J Neuroimmune Pharmacol 2012;7:1025-1033.

33. Soder HE, Berumen AM, Gomez KE, Green CE, Suchting R, Wardle MC, et al. Elevated neutrophil to lymphocyte ratio in older adults with cocaine use disorder as a marker of chronic inflammation. Clin Psychopharmacol Neurosci 2020;18:32-40.

34. Avcil S. Evaluation of the neutrophil/lymphocyte ratio, platelet/lymphocyte ratio, and mean platelet volume as inflammatory markers in children with attention-deficit hyperactivity disorder. Psychiatry Clin Neurosci 2018;72:522-530.

35. Pumariega AJ, Kilgus MD, Rodriguez L. Adolescence. In: Ruiz P, editor. Lewinsohn's textbook on addictions. Philadelphia: Lippincott Williams \& Williams;2005. p.1021-1037.

36. Kokkevi A, Kanavou E, Richardson C, Fotiou A, Papadopoulou $\mathrm{S}$, Monshouwer K, et al. Polydrug use by European adolescents in the context of other problem behaviours. Nord Stud A/cohol Drugs 2014;31:323-342.

37. Dir AL, Hulvershorn LA. Sex differences in adolescent neurobiological risk for substance use and substance use disorders. Curr Addict Rep 2019;6:514-521.

38. Fox HC, Sinha R. Sex differences in drug-related stress-system changes: implications for treatment in substance-abusing women. Harv Rev Psychiatry 2009;17:103-119.

39. Hilderbrand ER, Lasek AW. Studying sex differences in animal models of addiction: an emphasis on alcohol-related behaviors. ACS Chem Neurosci 2018;9:1907-1916.

40. Rainville JR, Hodes GE. Inflaming sex differences in mood disorders. Neuropsychopharmacology 2019;44:184-199. 
41. Attarzadeh-Yazdi G, Arezoomandan R, Haghparast A. Minocycline, an antibiotic with inhibitory effect on microglial activation, attenuates the maintenance and reinstatement of methamphetamine-seeking behavior in rat. Prog Neuropsychopharmacol Biol Psychiatry 2014;53:142-148.

42. Zhang L, Shirayama $Y$, Shimizu E, lyo $M$, Hashimoto K. Protective effects of minocycline on 3,4-methylenediox- ymethamphetamine-induced neurotoxicity in serotonergic and dopaminergic neurons of mouse brain. Eur I Pharmacol 2006; 544:1-9.

43. Sofuoglu M, Mooney M, Kosten T, Waters A, Hashimoto K. Minocycline attenuates subjective rewarding effects of dextroamphetamine in humans. Psychopharmacology (Berl) 2011;213:61-68. 